\title{
APROXIMACIÓN SISTÉMICA AL DIMENSIONAMIENTO DE PERSONAL EN INSTITUCIONES PRESTADORAS DE SERVICIOS DE SALUD
}

\author{
A system thinking approach to staff sizing in health care institutions
}

\author{
Julián Alberto Uribe-Gómez \\ MSc en Gestión Tecnológica. Esp en Estrategia gerencial y prospectiva. Facultad de Ciencias \\ Económicas y Administrativas, Instituto Tecnológico Metropolitano, Medellín-Colombia, \\ julianuribe@itm.edu.co
}

\section{Cómo citar / How to cite}

Uribe-Gómez, J. (2018). Aproximación sistémica al dimensionamiento de personal en instituciones prestadoras de servicios de salud. Revista CEA, 4(8), 51-65. https://doi.org/10.22430/24223182.1047

Recibido: 23 de enero de 2018

Aceptado: 7 de mayo de 2018

\section{Resumen}

El talento humano es la piedra angular en cualquier sistema de salud. Sin personal idóneo no es posible prestar un servicio de calidad a la población necesitada, por lo tanto, se hace indispensable contar con metodologías de trabajo que faciliten y fortalezcan la planeación y la gestión del personal de apoyo de los servicios de salud.

Este artículo pretende presentar un modelo de planeación de recursos utilizando la simulación, bajo el paradigma de dinámica de sistemas como herramienta de trabajo, con el objeto de brindar apoyo a aquellas personas encargadas de la elaboración de políticas y planes en instituciones de salud para tomar mejores decisiones, en lo referente a la cantidad de talento humano que deben contratar, para satisfacer la oportunidad, calidad y servicio en la atención.

Palabras clave: personal, metodología, simulación, cuidado de la salud, sistemas dinámicos, talento humano, planificación de recursos.

\begin{abstract}
Human talent is the cornerstone of any health system. Without suitable staff, a quality service cannot be provided to the needy. Therefore, methods that facilitate and improve the planning and management of the support staff in health services should be available.

Using simulations, this article presents a resource planning model that adopts the paradigm of system dynamics as a tool. The objective is to support the decision making process of those in charge of developing policies and plans at health care institutions regarding the human talent to hire in order to provide a timely, quality service.
\end{abstract}

Keywords: Staff, method, simulation, health care, system dynamics, human talent, resource planning.

\section{INTRODUCCIÓN}

Durante muchos años, las instituciones prestadoras de servicios de salud han experimentado dificultades consolidando las necesidades de personal médico requerido 
para una población general y específica y, al mismo tiempo minimizando el impacto en los costos (Linge, 2007). Sumado a esto, las instituciones tienen la responsabilidad de regular la oferta de servicios de salud para satisfacer la demanda que genere la población objeto. Por esto, es importante definir la capacidad de la oferta contratada por la institución, de tal forma que permita el acceso a los servicios de salud, de los afiliados, con características de calidad (Jurado \& Velásquez, 2009). Sin embargo, el personal médico representa un costo equivalente al $70 \%$ del gasto, por ello, los administradores deben asegurar su óptima disposición en la cantidad correcta, para alcanzar la máxima efectividad, calidad y equidad (Daviaud \& Chopra, 2008).

Una de las funciones primordiales de las instituciones de salud es la de organizar y garantizar, directa o indirectamente, la prestación del plan de salud obligatorio a los afiliados, así, los lineamientos normativos del sistema general de seguridad social en salud, permiten utilizar metodologías de planificación del talento humano de tipo cualitativo, entonces, cuando se integra la planificación estratégica de los recursos en la planificación de los negocios (Benjumea-Arias, Villa-Enciso, \& Valencia-Arias, 2016) que busca satisfacer las necesidades de los servicios de salud, es necesario contar con una metodología que permita cuantificar el talento humano en salud para cubrir las necesidades de acuerdo a unos parámetros o variables establecidas (Jurado \& Velásquez, 2009); principalmente los parámetros necesarios se encuentran dentro de la dinámica de la oferta y la demanda del sector salud, ya que con base en ellos se construyen y se soportan las relaciones necesarias para la construcción de un modelo cuantitativo y su futuro análisis.

Los centros de salud y hospitales se enfrentan a diversas dificultades como el incremento en los costos de los equipos, escasez de personal médico capacitado e infraestructura limitada. Con los costos de la salud aumentando, al igual que la población a atender, la industria de la salud está progresivamente enfrentada a dificultades de una demanda creciente y la disminución de sus reembolsos (Ballard \& Kuhl, 2006). Año tras año, las instituciones de salud esperan tratar más cantidad de pacientes de una manera más óptima en sus procesos, esto con el fin de captar mayor cantidad de recursos económicos destinados a la salud; por lo tanto, el talento humano es utilizado para contrarrestar la demanda que no coincide con las necesidades operacionales, por ello, es importante poder evaluar y planear cuantitativamente la red de servicios de salud (Jurado \& Velásquez, 2009).

Dentro de los servicios de salud, las variables capacidad y calidad están fuertemente ligadas, de hecho, como una unidad de medida de la calidad se tiene el indicador personal asistencial/paciente, el cual es comúnmente referido en la literatura sobre ciencias médicas (Trellevik, 2008), sin embargo, otras medidas de calidad para dichas instituciones serían por ejemplo, el tiempo promedio de espera en la institución o el estrés entre trabajadores asistenciales; sin embargo, estas medidas son cualitativas y relativas (Trellevik, 2008), lo que hace difícil su medición y posterior seguimiento. No obstante, muchas instituciones de salud miden la calidad con base en la métrica "oportunidad», la cual es entendida como:

La posibilidad que tiene el usuario de obtener los servicios que requiere, sin que se presenten retrasos que pongan en riesgo su vida o su salud. Esta característica se relaciona con la organización de la oferta de servicios en relación con la demanda y con el nivel de coordinación institucional para 
gestionar el acceso a los servicios. (Jurado \& Velásquez, 2009).

Esta medida se base en el tiempo de espera en días entre la solicitud de la cita a la institución de salud y la atención efectiva en consulta. La diferencia estándar es de dos días (Calisalud EPS, 2008) y cualquier atención que se realice por encima de este tiempo no es oportuna, es decir, no cumple con el pacto de calidad establecido.

A partir de estas premisas antes mencionadas, es válido abordar la siguiente pregunta de investigación:

¿Se puede plantear un modelo sistémico de planificación para atender la población afiliada de una institución prestadora de servicios de salud, de tal manera que se garantice la suficiencia de talento humano médico de forma eficiente y oportuna?

\section{MARCO TEÓRICO Y CONCEPTUAL}

Para modelar y analizar el sistema de salud se utilizara una aproximación sistémica mediante el paradigma de simulación de dinámica de sistemas, ya que tal y como explican los autores Borshchev y Filippov (2004), la economía de la salud pertenece a un nivel alto de abstracción, es decir, al nivel estratégico, es allí donde también se ubican la planeación de los recursos humanos; en la Figura 1 se pueden observar los principales paradigmas y sus niveles de abstracción.

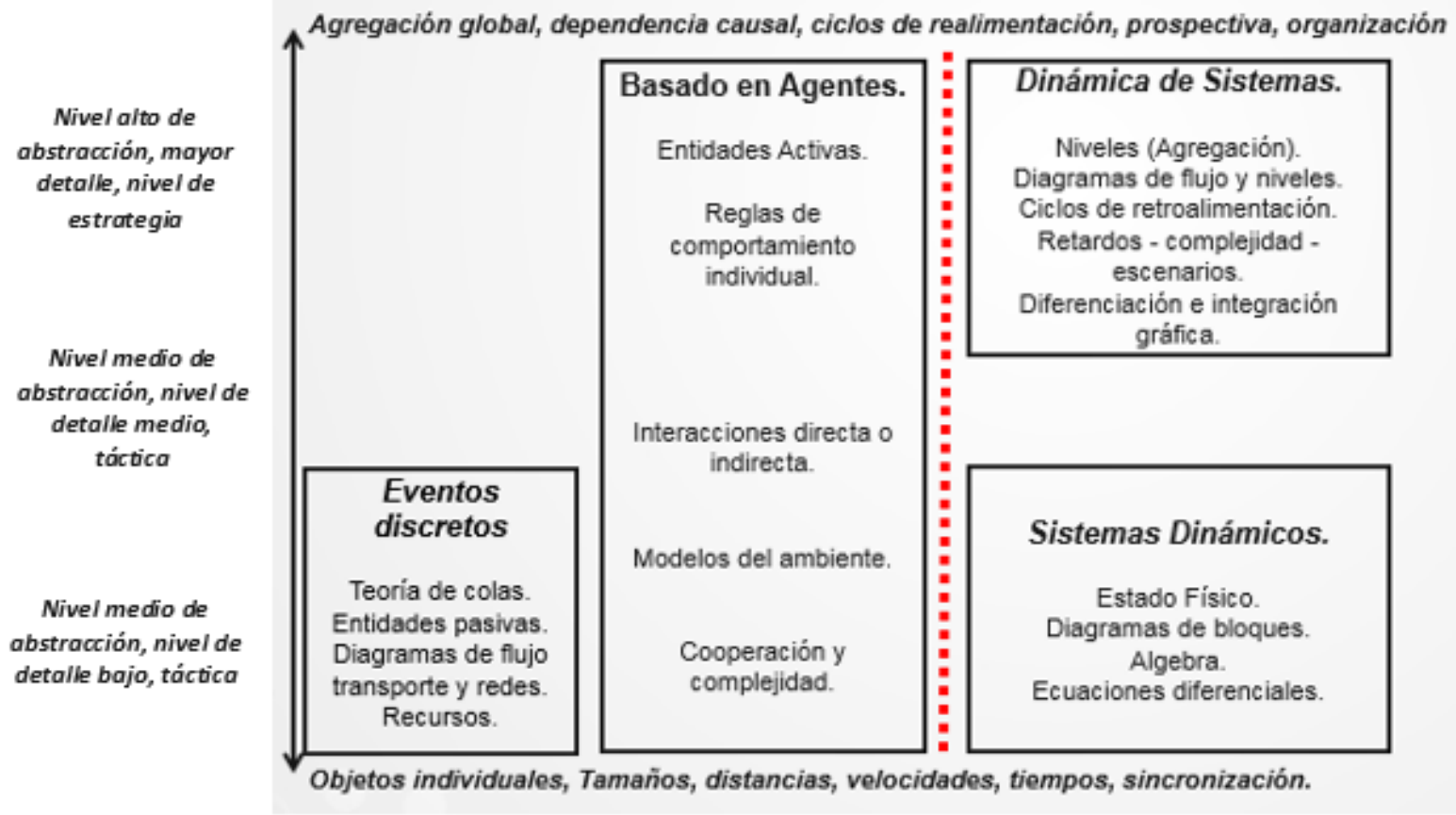

Figura 1. Paradigmas de simulación y niveles de abstracción

Figure 1. Simulation paradigms and abstraction levels Fuente: tomado de (Uribe, 2015).

De acuerdo con esto, la perspectiva sistémica, y más exactamente, la simulación es una técnica que permite crear modelos, apoyándose en programas informáticos, que posteriormente servirán para analizar el comportamiento de un sistema en diferentes 
circunstancias y generar destrezas en un ambiente controlado, analizando los posibles cambios, consecuencias y políticas. Desafortunadamente, aún son muchos los sectores productivos que no aprovechan las ventajas que esta herramienta metodológica ofrece para la toma de decisiones, el ahorro de costes o la optimización de procesos (Viveros y Chew, 2013 en Quintero \& Uribe, 2017).

Entendiendo esto, la Dinámica de Sistemas es definida por Sterman (2000) como: «La dinámica de sistemas es un método para mejorar el aprendizaje de los sistemas complejos». El sistema de salud y su planeación son considerados sistemas complejos debido principalmente a sus procesos, dimensiones y a su dinámica (Trellevik, 2008). La metodología implica el desarrollo de diagramas causales y modelos de simulación orientados a evaluar la estrategia y las políticas de las instituciones, a través de escenarios sistemáticos respondiendo a preguntas de la forma «Por qué» y «Qué pasaría sì.

El principio central de la dinámica de sistemas consiste en que el comportamiento complejo de los sistemas son el resultado de acumulaciones continuadas, ya sea de personas, materiales, información, etc., y de mecanismos de retroalimentación existentes. Por lo tanto, un modelo de dinámica de sistemas es una serie de ecuaciones diferenciales y algebraicas desarrolladas para ejemplificar el comportamiento del sistema; por lo tanto, la modelación es un proceso iterativo de generación de hipótesis, diagramación causal, cuantificación, simulación y análisis de políticas para la toma de decisiones.

De acuerdo con todo lo anterior, se justifica la visión sistémica y holística desde el contexto de la investigación y la propuesta, debido principalmente a que la modelación en dinámica de sistemas ha sido aplicada en temas de salud poblacional desde 1970, por ejemplo, para el análisis de capacidad de los sistemas de salud basados en el crecimiento poblacional, también en planeación, cuidado dental o salud mental (Homer \& Hirsch, 2006).

Por ello, resulta necesario entonces identificar y categorizar las variables más importantes para la construcción de la perspectiva sistémica, a partir de la economía de la salud. En la Tabla 1 se identifican las variables pertenecientes a la oferta y la demanda del servicio.

Tabla 1. Principales Variables de la Economía de Salud

Table 1. Main variables in health economics

\begin{tabular}{lll}
\hline & Dimensión de la economía de la salud & \\
\hline Oferta & Valor & Referencia \\
\hline Tiempo atención profesional & 20 minutos & (Ministerio de Salud, 1994) \\
Horas laborales semana profesional & 48 horas semanales & (Congreso de Colombia, 2017) \\
Días laborales semana profesional & 6 días semana & (Congreso de Colombia, 2017) \\
Semanas al mes & 4.33 semanas al mes & (Superfinanciera, 2007) \\
\hline Demanda & & \\
\hline Población a atender & $\begin{array}{l}\text { Depende del servicio y la } \\
\text { institución }\end{array}$ & (Calisalud EPS, 2008) \\
Frecuencia de uso & $\begin{array}{l}\text { Depende del servicio y la } \\
\text { institución }\end{array}$ & (Calisalud EPS, 2008) \\
\hline
\end{tabular}

Fuente: elaboración del autor con base en fuentes listadas en la tabla. 
En Colombia, el Ministerio de Salud (1994) establece en el Artículo 97, el tiempo de atención de la consulta médica, el cual no debe ser menor a 20 minutos, esto implica 3 pacientes por hora. El valor de la variable se presenta en (1).

(1) tiempo de atención médico $=20$ minutos $/$ paciente
Esto implica que la productividad en la atención de pacientes es 3 pacientes por hora, presentado en (2).

En ese orden de ideas, la capacidad de atención medico al día será definida como se encuentra mostrada en (4), si se tiene 8 horas laborales (3).

(2) Capacidad de atención médico $/$ hora $=\frac{60 \text { minutos } / \text { hora }}{20 \text { minutos } / \text { paciente }}=3 \frac{\text { pacientes }}{\text { hora }}$

(3) Horas laborales diarias $=8 \frac{\text { horas }}{\text { día }}$

(4) Capacidad de atención medico diaria

$$
=\text { capacidad de atención medico/hora } * \text { horas laborales diarias }=24 \frac{\text { pacientes }}{\text { dia }}
$$

Sin embargo, la literatura define que se asume que, en un día de 8 horas de trabajo, un profesional de la salud provee activamente servicio durante 6 horas y que 2 horas de su labor, están destinadas a otras actividades como reuniones, logística, administración o en tiempo de espera (Galaty \& Banda, 2005). De este modo la capacidad de atención medico diaria se reduciría.

Las particularidades que representa cada persona hacen de cualquier estudio en talento humano una labor un tanto compleja, no existen formulas que precisen sobre la mejor forma del como se debe comportar un empleado para obtener la mayor productividad en el desarrollo de sus responsabilidades (Franco-lópez \& Bedoya-Zapata, 2018), bajo esta premisa, Ango (2009) indica las actividades y las horas destinadas que no están relacionadas con el ejercicio de atención a usuarios, estas actividades tornan mas complicado el ejercicio de calcular con exactitud la productividad, dichas actividades se encuentran discriminadas en:

- Reuniones institucionales

- Capacitaciones

- Enfermedad

- Tiempo de descanso o Break

- Realizar informes

- Desplazamientos

De acuerdo con esto, tal como se muestra en la Tabla 2, se pueden percibir varios escenarios para calcular la capacidad de atención medica al mes, teniendo en cuenta las horas destinadas a actividades fuera de la operación. 
Tabla 2. Escenarios según la capacidad de atención de médicos al mes Table 2. Monthly health care capacity of physicians scenarios

\begin{tabular}{cccc}
\hline Escenario & $\begin{array}{c}\text { Pacientes } \\
\text { diarios }\end{array}$ & $\begin{array}{c}\text { Pacientes } \\
\text { semanales }\end{array}$ & $\begin{array}{c}\text { Pacientes } \\
\text { mensuales }\end{array}$ \\
\hline 8 horas laborales día & 24 & 144 & 623.52 \\
7 horas laborales día & 21 & 126 & 545.58 \\
6 horas laborales día & 18 & 108 & 467.64 \\
\hline
\end{tabular}

Fuente: elaboración propia.

De otro lado, Jurado y Velásquez (2009), presentan una perspectiva de cálculo de pacientes mensuales de 528 pacientes, teniendo en cuenta 22 días laborales por mes para el profesional de la salud. Esto equivaldría a estimar una improductividad del $10 \%$ en las actividades diarias, las cuales estan destinadas a otros oficios diferentes a la actividad asistencial. Por lo tanto, las horas laborales diarias son equivalentes al resultado presentado en (5).

Lo que genera una capacidad de atención mensual calculada en (6):
Por otro lado, al estimar el número de días efectivos laborales en un mes, se recurre a tomar los días anuales, restándole los festivos y dominicales, obteniendo así los días laborales al mes; ahora bién, al promediar los datos de 6 años, como se observa en la Tabla 3, se puede obtener un estimativo que puede ser utilizado en el cálculo de los pacientes que atenderá el médico mensualmente.

Hagopian et al. (2012) estiman el número de horas trabajadas al año para los profesionales, multiplicando los días trabajados por el número de horas al día, restando los días festivos, tiempos de entrenamiento, enfermedad y vacaciones.

(5) Horas laborales diarias $=7.2 \frac{\text { horas }}{\text { día }}$

(6) Capacidad de atención medico diaria

$$
=\text { capacidad de atención medico hora } * \text { horas laborales diarias }=2
$$

Tabla 3. Días Laborales Efectivos Mensual

Table 3. Business days

\begin{tabular}{cccccc}
\hline Año & Días al año & Festivos & Domingos & Días laborables año & Días laborables mes \\
\hline 2012 & 366 & 20 & 53 & 293 & 24.41 \\
2013 & 365 & 20 & 52 & 293 & 24.41 \\
2014 & 365 & 19 & 52 & 294 & 24.5 \\
2015 & 365 & 20 & 52 & 293 & 24.41 \\
2016 & 366 & 19 & 52 & 295 & 24.58 \\
2017 & 365 & 19 & 52 & 294 & 24.5 \\
& & & & Promedio & 24.46 \\
\hline
\end{tabular}

Fuente: elaboración propia. 
Por lo tanto, al hacer el cálculo del número de pacientes médicos al mes se obtiene el resultado presentado en (7):

\section{Calculo de demanda del servicio}

La demanda es una de las principales variables consideradas en la teoría económica, dado que, a partir de su definición y medida, muchos analistas realizan estimaciones de otras variables relacionadas. En el sistema de salud, como cualquier otro, existen flujos de demanda y oferta que buscan equilibrar la capacidad del sistema y optimizarlos.

Por lo tanto, la demanda estimada puede ser definida como la población afiliada a la institución multiplicada por la frecuencia de uso del servicio (Calisalud EPS, 2008), de este modo la ecuación que la caracteriza es la presentada en (8).

Otra noción más simple indica que la demanda del servicio es el número total estimado de pacientes que requieren el servicio (Hagopian et al., 2012).

Los clientes se evalúan en función de sus demandas o necesidades actuales y futuras, incluyendo el uso que se pretende (OrtizDelgadillo, Esquivel-Aguilar, \& HernándezCastorena, 2017). Además, otro componente importante de la demanda es el número de consultas que realiza un usuario al servicio, esto se denomina la frecuencia de uso del servicio y será definida como el número de atenciones promedio por cada usuario afiliado durante un periodo de tiempo (Calisalud EPS, 2008) o el número de veces que la actividad se realiza en un periodo de tiempo determinado (Ango, 2009). Donde, en promedio para el servicio de consulta médica, la frecuencia estimada es de 2.3 consultas-médico al año (Calisalud EPS, 2008).

La demanda como factor principal de la necesidad de servicios, es en muchos sentidos una variable altamente dinámica, ya que existen muchos factores que generan fluctuaciones sobre su valor y hacen difícil una estimación acertada, algunos factores a tener en cuenta según Trellevik (2008) son:

- Población actual por edad y género

- Patrones migratorios

- Fertilidad y nacimientos

- Mortalidad en todos los grupos de edades

La magnitud de la demanda de los servicios es entonces, entendida como una dinámica compleja de la población, esto se puede observar en la Figura 2.

\section{(7) Pacientes medico mensual}

$=$ Promedio dias laborables mes $*$ capacidad de atención medico diaria

$=528.33$ pacientes medico mensual

(8) Demanda Estimada = Población afiliada $*$ Frecuencia de uso del servicio 


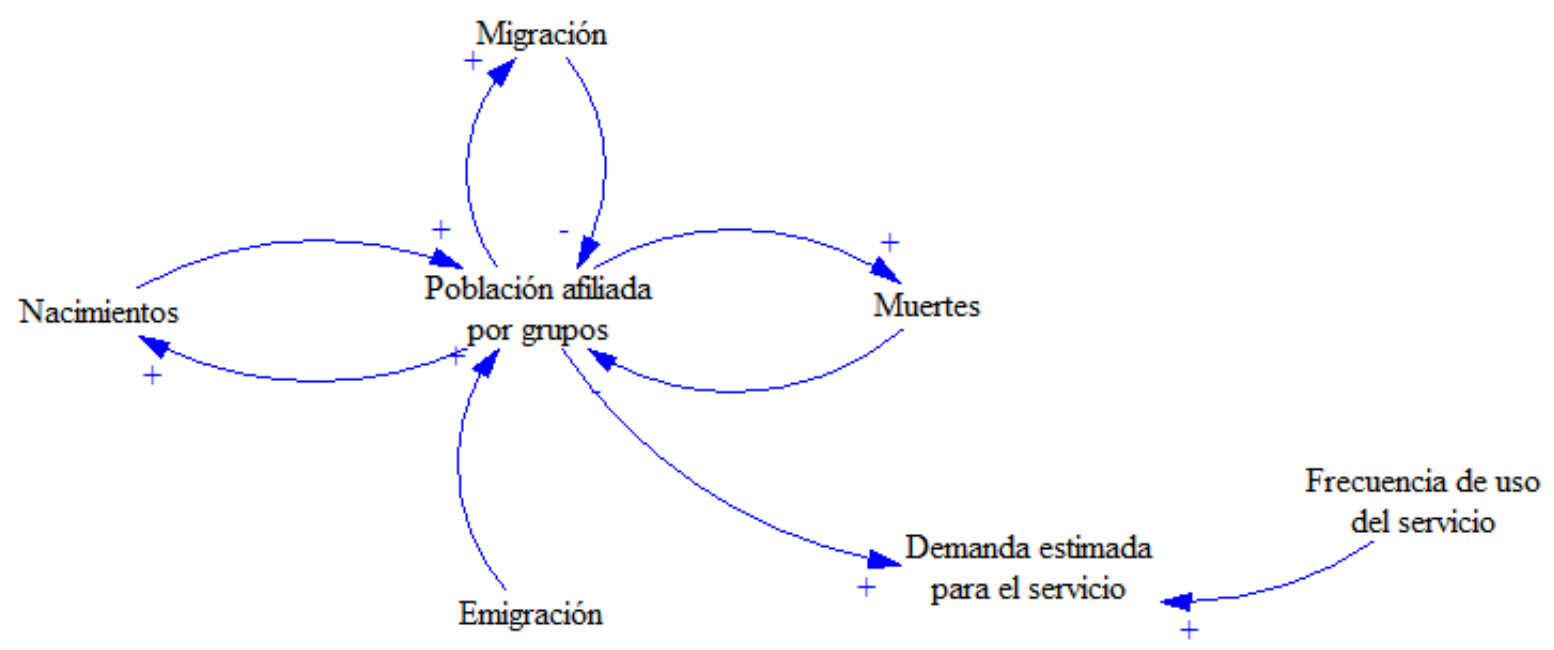

Figura 2. Perspectiva de la dinámica de la demanda

Figure 2. Overview of the dynamics of demand

Fuente: elaboración propia.

\section{Cálculo de oferta del servicio}

Una vez definidas las necesidades y el método de cálculo y evaluación de la demanda, resulta imprescindible generar balance en el sistema, por lo tanto, se debe estimar la oferta. Muchos de los planificadores de la fuerza laboral para salud utilizan combinaciones de indicador de médicos por población, patrones históricos en formas de series temporales y el juicio de los profesionales de la salud, para estimar la oferta de los servicios que satisfagan la oferta. Otros análisis más sofisticados utilizan cálculos de la fuerza laboral, a través de teoría de colas y líneas de espera, funciones de producción o análisis de regresión (Hagopian et al., 2012). Dentro de la revisión de literatura, frente a este tema se encontraron algunos cálculos para

estimar esta oferta. Un ejemplo particular es el estimado de fuerza laboral para la demanda de pacientes en un servicio de psiquiatría, este se ha estimado mediante la ecuación (9) (Hagopian et al., 2012):

(9) Numero de psiquiatras requeridos $=\frac{(\text { Número de pacientes que necesitan atención } * \text { Cantidad de tiempo requerido para tratamiento })}{\text { Cantidad de tiempo ofrecido por psiquiatra }}$

Otras fuentes proponen calcular las necesidades de médicos sobre la carga de trabajo y los tiempos medios de atención (Ministerio de Sanidad y Politica Social, 2010), la forma de cálculo es la ecuación (10).

Sin embargo, debido a que la oferta del servicio se encuentra ligada a la productividad del personal y al número de personas que se encuentran en el servicio, teniendo en cuenta que este personal es fluctuante y dinámico, debido a los efectos de las políticas de contrataciones y retiros que existen en toda institución, una relación válida para esta variable se puede ver en la Figura 3.

(10) Médicos requeridos

$$
=\frac{\text { media del } \% \text { horario que consume un paciente } * \# \text { de asistencias al año }}{\# \text { de horas anuales contratadas por médico }}
$$




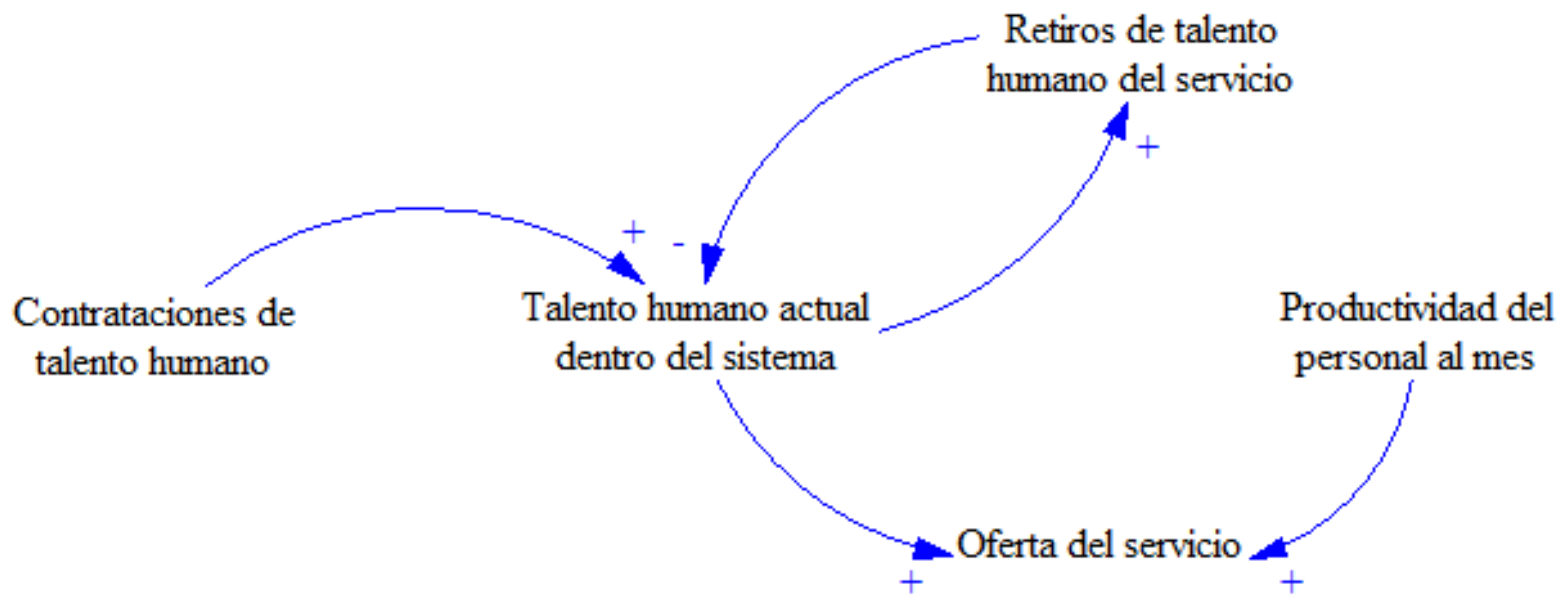

Figura 3. Perspectiva de la oferta de los servicios

Figure 3. Overview of the dynamics of service offer Fuente: elaboración propia.

\section{Calculo de brechas o superávits del servicio}

Diversos autores han explorado patrones para considerar cual debe ser el número ideal de personal médico para una demanda específica, estos estándares son:

- 5.63 médicos generales en una población de 5000 habitantes (Facultad Nacional de Salud Pública, 1965).

- 50,2 médicos para una población de 100000 pacientes (Health Leaders, 2009).

- 1 médico de tiempo completo por cada 4000 pacientes (Ministerio de Sanidad y Politica Social, 2010).

Sin embargo, los estándares mencionados se quedan cortos, debido a la dinámica que existe dentro del sistema y también a otros factores de interés, que pueden afectar a los profesionales de la salud requeridos (Health Leaders, 2009), estos son:

- Envejecimiento de la población, que afecta a indicador médicos por población.

- Crecimientos o decrecimientos proyectados de la población.
- Incrementos en diferentes especialidades.

- Retiros o renuncias de médicos.

- Poca actividad de médicos a punto de retirarse.

- Mucha más actividad de médicos sin experiencia, tratando de aprender.

Debido a esto, la literatura ha recurrido a calcular los requerimientos con base en las brechas o los superávits, principalmente Hagopian et al. (2012) propone determinar la fuerza de trabajo requerida para llenar la brecha o el superávit, tomando la demanda y restando la oferta del servicio, esto se divide por las horas de servicio a tiempo completo que trabajaría un profesional; posteriormente, se calcula el indicador oferta sobre demanda para estimar un parámetro comparable a la magnitud calculada con la brecha o el superávit. Si este indicador es equivalente a 1 se espera un balance perfecto entre variables, menor que 1 indica brechas y mayor que 1 indica superávit (Hagopian et al., 2012).

La relación entre las variables demanda y oferta para indicar este balance, se puede denominar eficiencia. La medición de la 
eficiencia o rendimiento en las organizaciones, se está convirtiendo cada vez más en un elemento clave para la competitividad y de diferenciación entre instituciones (OrtizDelgadillo et al., 2017). Este se puede visualizar, tal y como se propone en la Figura 4.

La eficiencia establecida es un indicador que ayuda a identificar si existen brechas 0 superávits; ahora, al conocerse el número de profesionales médicos disponibles para atender el servicio y el número de profesionales médicos requeridos para prestar el servicio, se puede calcular la magnitud de la brecha en el número de profesionales, como la diferencia entre los profesionales disponibles y los requeridos (Galaty \& Banda, 2005), esta relación se puede observar en la Figura 5.

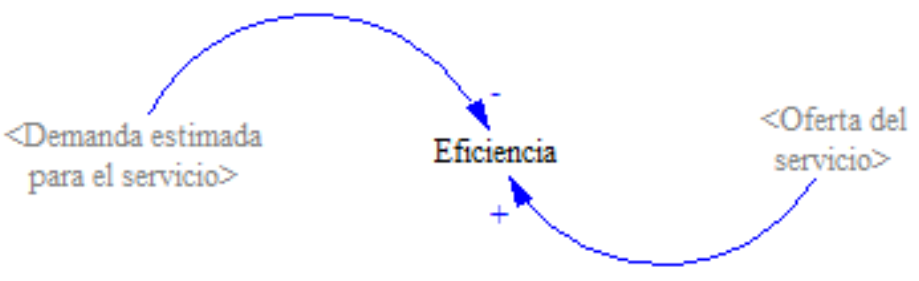

Figura 4. Relación entre la oferta y la demanda

Figure 4. Relationship between supply and demand Fuente: elaboración propia.

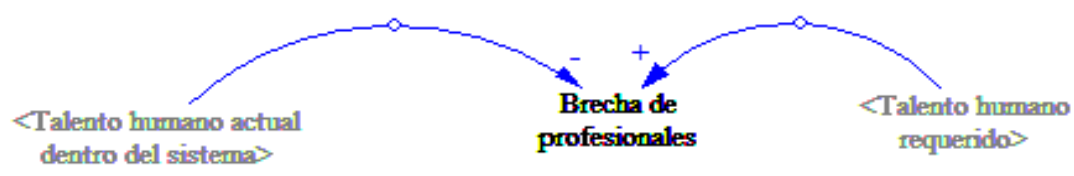

Figura 5. Relación para el cálculo de la brecha de profesionales

Figure 5. Relationship for calculating the professional practice gap Fuente. elaboración propia.

\section{RESULTADOS Y DISCUSIÓN}

Una vez se han definido las variables principales que afectan el sistema y el servicio, se procede a sintetizarlo y a relacionar los diferentes ítems de estudio en el diagrama causal presentado en la Figura 6. A partir de este diagrama se puede estudiar el comportamiento del sistema y estudiar las variables más significativas; en este orden, el modelo de simulación está diseñado para ayudar a un centro de salud u hospital a evaluar su eficiencia actual y como los cambios en los recursos o en la demanda pueden afectar su desempeño (Ballard \& Kuhl, 2006). Además, el modelo de simulación es generalizado, esto implicaría que cualquier centro podría realizar sus propios análisis determinando su eficiencia y desempeño.

Ahora bien, suponga que una institución tiene los siguientes parámetros de demanda, como se observa en la Tabla 4 y la Figura 7. 


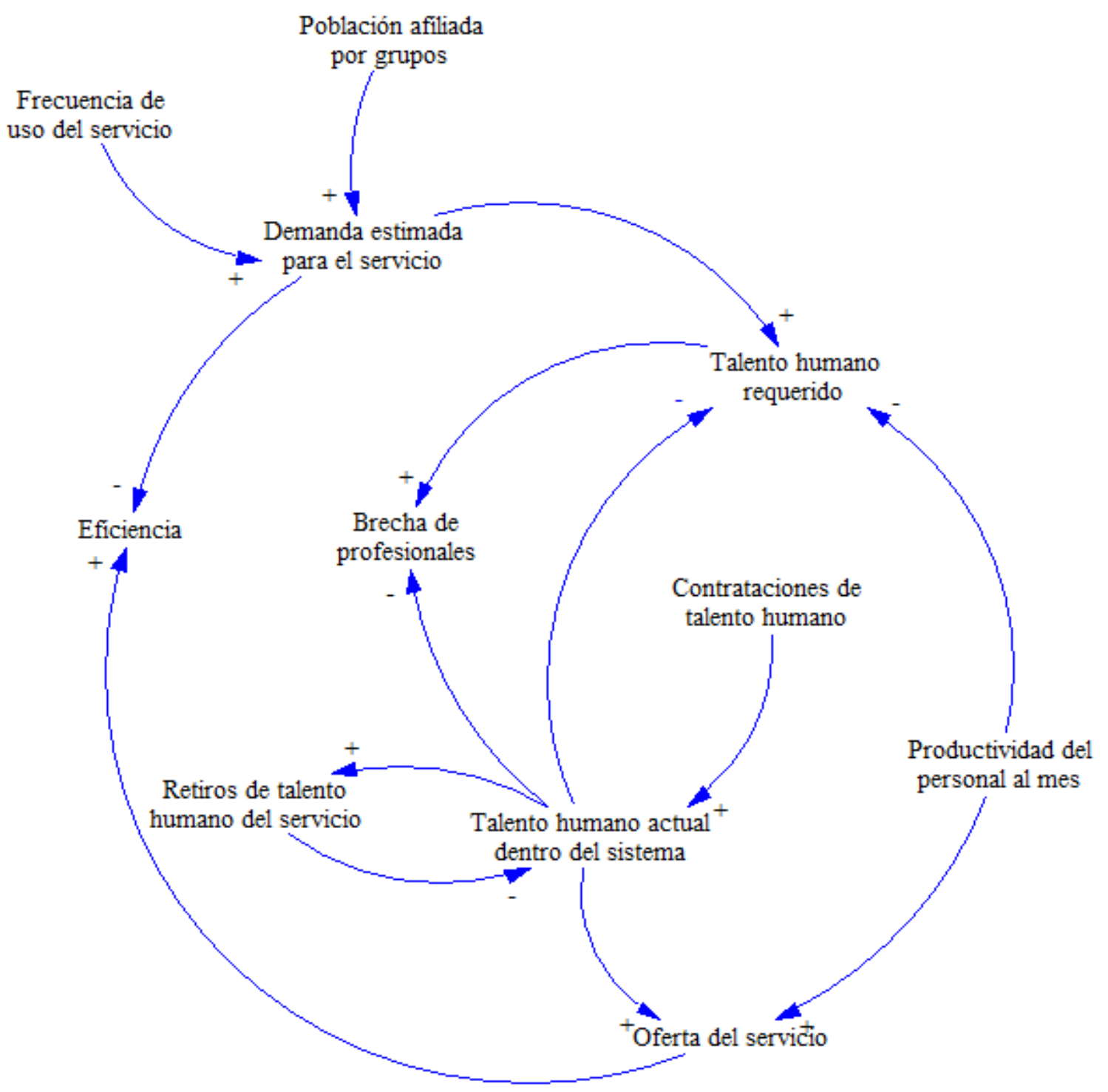

Figura 6. Dinámica y relaciones entre las variables del servicio de salud

Figure 6. Dynamics and relationships between variables in health care services Fuente: elaboración propia.

Tabla 4. Valores supuestos para el cálculo de la demanda Table 4. Values assumed to calculate the demand

\begin{tabular}{ll} 
Variables para la demanda & Valor para el cálculo \\
\hline Población afiliada & 50000 \\
Frecuencia de uso del servicio & 2 \\
\hline
\end{tabular}

Fuente: elaboración propia. 


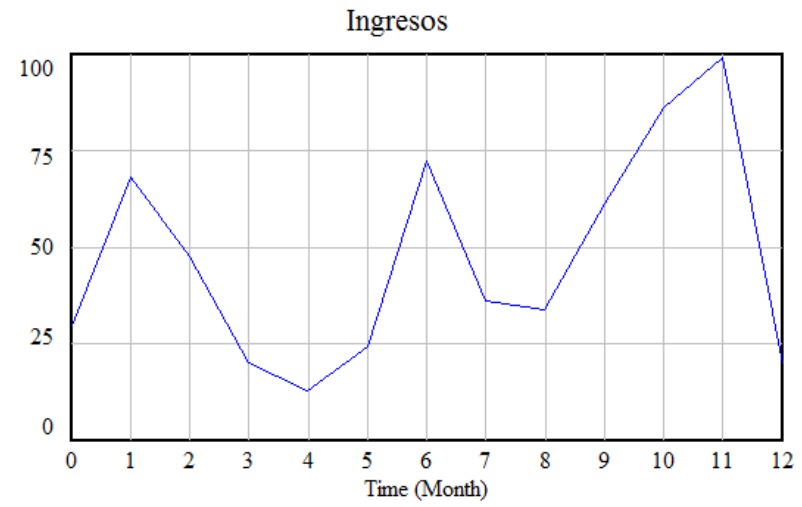

Figura 7. Comportamiento de los ingresos de afiliados durante 1 año

Figure 7. Number of new enrollees in one year Fuente: elaboración propia.

Se espera que la demanda se comporte de forma dinámica debido al comportamiento de las variables; si se simulan 12 meses entonces el valor de la población y la demanda serán los presentados en la Tabla 5.
Para el cálculo del componente de la oferta del servicio, se utilizarán los valores de las variables presentados en la Tabla 6.

Tabla 5. Demanda del servicio

Table 5. Service demand

\begin{tabular}{rrr}
\multicolumn{1}{l}{ Mes } & Demanda & Población \\
\hline 0 & 8333.33 & 50000 \\
1 & 8345.54 & 50073.3 \\
2 & 8364.06 & 50184.4 \\
3 & 8372.96 & 50237.7 \\
4 & 8382.6 & 50295.6 \\
5 & 8388 & 50328 \\
6 & 8392.37 & 50354.2 \\
7 & 8406.07 & 50436.4 \\
8 & 8413.17 & 50479 \\
9 & 8422.74 & 50536.4 \\
10 & 8440.33 & 50642 \\
11 & 8455.44 & 50732.6 \\
12 & 8474.77 & 50848.6 \\
\hline
\end{tabular}

Fuente: elaboración propia.

Tabla 6. Variables para la oferta del servicio

Table 6. Variables for service provision

\begin{tabular}{ll}
\hline Variables & Valores \\
\hline Días al mes & 24.46 días al mes \\
Horas al día & 8 horas al día \\
Productividad hora & 3 pacientes hora \\
Improductividad & $10 \%$ \\
Productividad & 528.33 pacientes medico al mes \\
\hline
\end{tabular}


Pero, suponiendo que el talento humano actual es de 12.5 profesionales, tal y como sugiere el Ministerio de Sanidad y Política Social (2010), de acuerdo con el estándar de 1 profesional por cada 4000 pacientes, se tiene entonces que la oferta, la eficiencia y el talento humano requeridos para el sistema son los presentados en la Tabla 7.

Tabla 7. Oferta del servicio y resultados de eficiencia y talento requerido Table 7. Service offer, efficiency, and required human talent

\begin{tabular}{rrrr}
\hline Mes & Oferta del servicio & Eficiencia & Talento Humano Requerido \\
\hline 0 & 6604.2 & 0.792504 & 1.26 \\
1 & 6604.2 & 0.791344 & 1.26 \\
2 & 6604.2 & 0.789592 & 1.26 \\
3 & 6604.2 & 0.788747 & 1.26 \\
4 & 6604.2 & 0.787847 & 1.26 \\
5 & 6604.2 & 0.787339 & 1.27 \\
6 & 6604.2 & 0.786929 & 1.27 \\
7 & 6604.2 & 0.785647 & 1.27 \\
8 & 6604.2 & 0.784984 & 1.27 \\
9 & 6604.2 & 0.784091 & 1.27 \\
10 & 6604.2 & 0.782458 & 1.27 \\
11 & 6604.2 & 0.781059 & 1.28 \\
12 & 6604.2 & 0.779278 & 1.28 \\
\hline \multicolumn{3}{c}{ Fuente: elaboración propia. }
\end{tabular}

Bajo los mismos parámetros, al buscar la óptima asignación de recursos del sistema, es decir con eficiencia equivalente a 1 , se evidencia que el número de talento humano dentro del sistema debe ser equivalente aproximadamente a 16 .

\section{CONCLUSIONES}

La perspectiva sistémica permite indagar sobre las relaciones de las variables de forma más holística y global, analizando la estrategia sobre cada una de ellas.

Por otro lado, al analizar los resultados, se puede notar que la eficiencia es mayor de 1 , lo que indica que hay un superávit, es decir, existe más talento humano, lo que puede impactar negativamente los costos del personal. Sin embargo, al tener uno resultado mayor que 1 implica que se está garantizando la suficiencia en el talento humano de la institución.

Además, el método permite, de manera ecuacional y cuantitativa, evidenciar que existen estándares que sobre dimensionan o sub dimensionan el talento humano; y como se ha aclarado, esto impacta en las gestiones financieras y laborales de las instituciones de salud.

Es importante conocer y estandarizar los roles que los profesionales desarrollan en las instituciones, debido a que muchos de estos se asumen como disponibles para la atención de los pacientes; sin embargo, se encuentran actuando en un rol administrativo, por lo tanto, no atienden pacientes ni tienen un rol operativo, lo cual subestima el número de profesionales disponibles y necesarios para la atención. 
El modelo es genérico y se presta para muchas extensiones de servicios de salud, no solo para el modelo de medicina general presentado, sino también para otros servicios como odontología, enfermería, servicios especializados, psiquiatría, entre otros.

\section{REFERENCIAS}

Ango, J. (2009). Propuesta metodológica para la estimación de recursos humanos del equipo básico de salud del primer nivel de atención en el marco del aseguramiento universal y la implementación del listado priorizado de intervenciones sanitarias.

Ballard, S. M., \& Kuhl, M. E. (2006). The use of simulation to determine maximum capacity in the surgical suite operating room. Proceedings - Winter Simulation Conference, 433-438. doi: 10.1109/WSC.2006.323112

Benjumea-Arias, M. L., Villa-Enciso, E. M., \& Valencia-Arias, J. (2016). Beneficios e impactos del teletrabajo en el talento humano. Resultados desde una revisión de literatura. Revista CEA, 2(4), 59-73. doi: $10.22430 / 24223182.172$

Borshchev, A., \& Filippov, A. (2004). From System Dynamics and discrete event to practical Agent Based Modelling: reasons, techniques, tools (p. 23).

Calisalud EPS. (2008). Manual de suficiencia de la red de prestadores de servicios de salud.

Congreso de Colombia. (2017). Ley 1848. Recuperado de http://es.presidencia.gov.co/normativa/ normativa/LEY 1846 DEL 18 DE JULIO DE 2017.pdf
Daviaud, E., \& Chopra, M. (2008). How much is not enough? Human resources requirements for primary health care: $A$ case study from South Africa. Bulletin of the World Health Organization, 86(1), 4651. doi: 10.2471/BLT.07.042283

Facultad nacional de salud pública. (1965). Metodología para el cálculo de recurso humano en salud según planes de beneficios, perfil epidemiológico, demanda histórica y nuevos roles.

Franco-lópez, J. A., \& Bedoya-Zapata, J. (2018). Análisis de modelos del talento humano aplicados en organizaciones pymes Revista CEA, 4(7), 85-101. Recuperado de http://revistas.itm.edu.co/index.php/revi sta-cea/article/view/761

Galaty, D., \& Banda, P. (2005). Human resources crisis in the Zambian health system : A call for urgent action. Tropical Medicine.

Hagopian, A., Mohanty, M. K., Das, A., \& House, P. J. (2012). Applying WHO's "workforce indicators of staffing need" (WISN) method to calculate the health worker requirements for India's maternal and child health service guarantees in Orissa State. Health Policy and Planning, 27(1), 11-18. doi: 10.1093/heapol/czr007

Health Leaders. (2009). How Many Physicians Make a Health System? In The Hospital Executive's Guide to Physician Staffing. Recuperado de http://www.healthleadersmedia.com/su pplemental/6693_browse.pdf

Homer, J. B., \& Hirsch, G. B. (2006). System dynamics modeling for public health: Background and opportunities. American Journal of Public Health, 96(3), 452-458. doi: 10.2105/AJPH.2005.062059 
Jurado, L., \& Velásquez, Y. (2009). Análisis de Suficiencia de Red de Servicios de Baja Complejidad en el Departamento de Nariño de la EPS-1 Mallamas.

Linge, C. E. . (2007). Patient flow through the central surgery unit at Haukeland Universitetssykehus. University of Bergen.

Ministerio de salud. (1994). Resolución 5261 de 1994.

Ministerio de Sanidad y Politica Social. (2010). Unidades de Urgencias Hospitalarias. Estándares y recomendaciones. Informes, Estudios Y Publicaciones, 1-148. Recuperado de http://www.msssi.gob.es/organizacion/s ns/planCalidadSNS/docs/UUH.pdf

Ortiz-Delgadillo, G., Esquivel-Aguilar, E. O., \& Hernández-Castorena, O. (2017). El impacto de la relación con el cliente y de la capacidad de valor agregado en el servicio en el rendimiento de la Pyme Manufacturera en Aguascalientes. Revista CEA, 2(4), 47-58. doi: 10.22430/24223182.171

Sterman, J. D. (2000). Business Dynamics: Systems Thinking and Modeling for a
Complex World. McGraw-Hill/Irwin.

Superfinanciera. (2007). Pensión de vejez, régimen de transición, ingreso base de liquidación, monto. Recuperado de https://www.superfinanciera.gov.co/SFC ant/Normativa/Conceptos2007/2006052 332.pdf

Trellevik, L. K. . (2008). Planning for an elderly boom; A System Dynamics Approach to strategic healthcare planning in Bergen, Norway. University of Bergen.

Uribe, J. A., \& Quintero, S. (2017). Aplicación de los modelos de simulación en entronos productivos bajo la metodología de teoría de las restricciones. Revista CEA, 3(6), 22. doi: 10.22430/24223182.654

Uribe Gómez, J. A. (2015). Análisis de la estructura, relaciones y dinámicas de los agentes del sistema regional de innovación en Antioquia. Universidad Pontificia Bolivariana.

Viveros, L., \& Chew, M. (2013). Entrenamiento para toma de decisiones por medio de simulación y casos de estudio. Revista Internacional de La Educación En Ingenieria, 6(1), 1-7. 